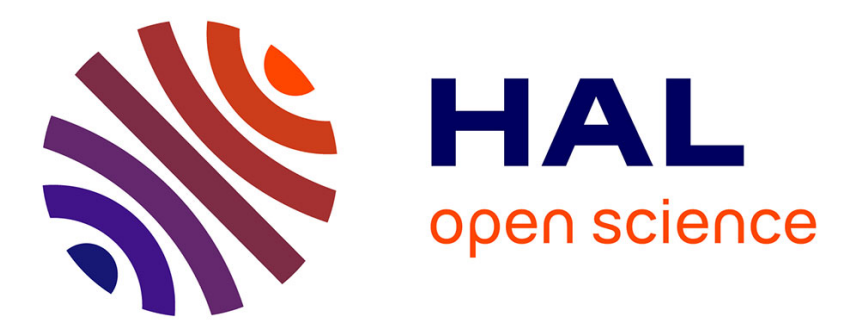

\title{
Digestibilité et activité biochimique intracæcale chez le poney recevant un même aliment complet présenté sous forme granulée, expansée ou semi-expansée
}

R. Wolter, D. Gouy, Andrée Durix, J. C. Letourneau, Monique Carcelen, Jacqueline Landreau, A. Bruny, A. Villard

\section{To cite this version:}

R. Wolter, D. Gouy, Andrée Durix, J. C. Letourneau, Monique Carcelen, et al.. Digestibilité et activité biochimique intracæcale chez le poney recevant un même aliment complet présenté sous forme granulée, expansée ou semi-expansée. Annales de zootechnie, 1978, 27 (1), pp.47-60. hal-00887788

\section{HAL Id: hal-00887788 https://hal.science/hal-00887788}

Submitted on 1 Jan 1978

HAL is a multi-disciplinary open access archive for the deposit and dissemination of scientific research documents, whether they are published or not. The documents may come from teaching and research institutions in France or abroad, or from public or private research centers.
L'archive ouverte pluridisciplinaire HAL, est destinée au dépôt et à la diffusion de documents scientifiques de niveau recherche, publiés ou non, émanant des établissements d'enseignement et de recherche français ou étrangers, des laboratoires publics ou privés. 
Ann. Zootech., I97\$, 27 (I), 47-60.

\title{
Digestibilité et activité biochimique intracæcale chez le poney recevant un même aliment complet présenté sous forme granulée, expansée ou semi-expansée
}

\author{
R. WOLTER, D. GOUY, Andrée DURIX, \\ J. C. LETOURNEAU, Monique CARCELEN \\ et Jacqueline LANDREAU, \\ avec la collaboration technique de A. BRUNy et A. VIL,ARD \\ Laboratoire de Nutrition et d'Alimentation, \\ École Nationale Vétérinaive de Lyon, \\ Marcy l'Étoile, 6926o Charbonnières (France)
}

\section{Résumé}

In même alinent complet, dosant $16,5 \mathrm{p}$. cent de cellulose et to p. cent de protéines (tabl. I) et marqué arec 0,2 p. cent d'oxyde de chrome, est présenté sous forme granulée, expansée ou semi-expansée (seule la partie cérśalière subit alors le traitement hydrothermique). Il est distribué :

- d'une part, à 6 poneys en cages à digestibilité, à raison de $3 \mathrm{~kg} / \mathrm{Al} / \mathrm{j}$, répartis en 2 repas:

- d'autre part, à 3 poneys munis d'une fistule permanente du cæcum et ne recevant que $2 \mathrm{~kg} / \mathrm{Al} / \mathrm{j}$, partagés en 2 repas d'une heure.

Dans ces conditions d'un régime strictement restreint, fractionné et relativement concentré, il n'apparaît pas de différences importantes entre les 3 présentations de l'aliment quant à la digestibilité totale (tabl. 2), à la digestibilité partielle atteinte au niveau du cæcum (tabl. 5), au métabolisme intracæral (tabl. 7) (jugé à travers le $\mathrm{pH}$ et les taux d'ammoniac, d'acides gras volatils, d'acide lactique) (fig. I, $2,3,4$ ) pas plus qu'en ce qui concerne la glycémie et la lactacidémie (tabl. 3).

A l'occasion de ces mesures de digestibilité, nous avons observé que le recours au traceur naturel représenté par la lignine, dosée selon la méthode de Van Soest, était nettement plus fiable que le marquage de l'aliment avec de l'oxyde de chrome (tabl. 4).

De façon générale, il ressort qu'environ $80 \mathrm{p}$. cent des matières sèches et des substances azotées digestibles sont résorbées dès l'intestin grêle dont le rôle est sans doute privilégié par ce mode d'alimentation. Dans le cæcum, les fermentations, qui ne disposent alors que d'un substrat épuisé, essentiellement cellulosique, restent modérées dans l'ensemble mais avec un regain d'activité 5 à 8 heures après le repas, alors qu'arrive le maximum de la phase particulaire; elles entraînent seulement un léger abaissement du $\mathrm{pH}$, vers 6,7 au lieu de 7 , à la faveur du doublement du taux des acides gras volatils dont les proportions demeurent inchangées, alors que la teneur en acide lactique reste très basse. 


\section{Introduction}

Au cours d'essais précédents, nous avons étudié la digestion chez les équidés, soit par des mesures de digestibilité et de vitesse du transit digestif, concernant notamment un foin long, broyé ou aggloméré (WOLTER, DURIX et LETOURNEAU, I974 et 1975) ainsi qu'un aliment complet, en farine, en granulés ou expansé (Wolter, DURIX et Letourneau, 1976), soit par analyse du contenu des différents segments du tube digestif après abattage (WoLTER et Gouy, 1976; Gour, 1976). Cette dernière technique qui donne une bonne image de la digestion, mais seulement à un moment donné par rapport au repas, mérite d'être complétée par la mise à profit d'animaux porteurs de canules permanentes"du tube digestif, permettant un examen cinétique de la digestion en des points privilégiés.

Ainsi, dans le cas présent, nous comparons 3 présentations (granulée, expansée ou semi-expansée), d'un aliment complet, qui est distribué :

- d'une part, à 6 poneys en cages à métabolisme, dans le but de déterminer les coefficients de digestibilité totale, ainsi que les taux sanguins de glucose et d'acide lactique;

- d'autre part, à 3 poneys munis de fistules permanentes du cacum, au niveau duquel est évaluée la digestibilité partielle, en comparaison avec la digestibilité totale mesurée chez ces mêmes sujets; en outre, l'évolution du contenu cæcal est suivie quant au $\mathrm{pH}$ et aux taux d'ammoniac, d'acides gras volatils et d'acide lactique.

\section{Matériel et méthodes}

\section{A. - Essais sur 6 poneys en cages à métabolisme}

\section{I. - Animaux.}

Selon le protocole habituellement mis en cuvre (Wolter, Durix et LETourNEAU, 1976) 6 poneys mâles de $200 \mathrm{~kg}$ de poids moyen, sont placés en cages à métabolisme. Ils reçoivent, au cours de 3 périodes de 3 semaines $(2$ semaines d'adaptation, I semaine expérimentale), le même aliment complet présenté alternativement sous forme granulée, expansée ou semi-expansée.

Chaque animal est rationné à $3 \mathrm{~kg} / \mathrm{j}$, répartis en 2 repas égaux, distribués à 8 et $I 7$ heures. L'essai a été répété 2 fois et les résultats exprimés sont les moyennes des 2 périodes expérimentales.

\section{2. - Aliments.}

La totalité de la farine de l'aliment expansé subit le traitement hydrothermique alors que, pour l'aliment semi-expansé, seule la fraction céréalière est traitée. Ce dernier se présente alors comme un mélange hétérogène de gros flocons blancs et de petits granulés verts comportant notamment la portion fourragère, les minéraux et les vitamines. L'expansion elle-même est réalisée au moyen d'un expandeur "Extapro" (presse Anderson) comme nous l'avons précisé dans une publication précédente (Woiter, Durix, Letourneau, I975).

Le tableau I récapitule la composition centésimale des aliments. 
TABIEAU I

Composition des aliments (en p. cent)

Composition of experimental diets

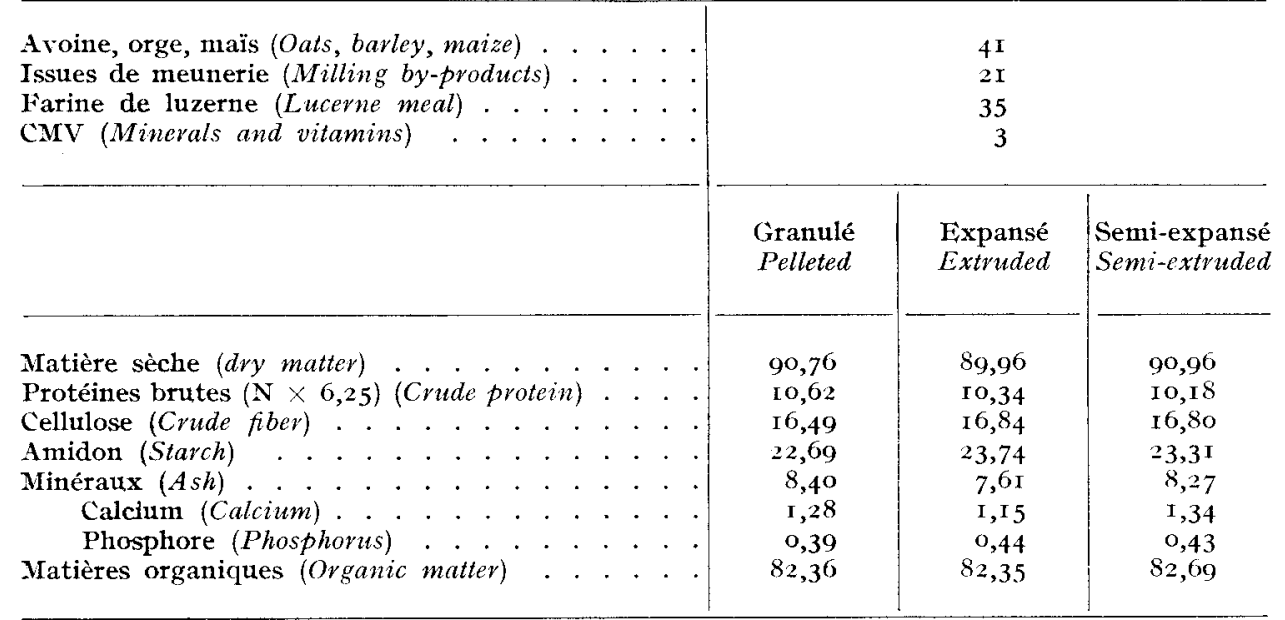

\section{3. - Techniques de laboratoire.}

Les aliments et les fèces sont soumis aux dosages des principaux constituants a fin de calculer leurs coefficients d'utilisation digestive totale. Les protéines brutes sont évaluées par la méthode de $\mathrm{KJELDAHL}(\mathrm{N} \times 6,25)$. La cellulose brute est estimée par la méthode de Scharrer (Scharrfr et Kurschner, I932) qui consiste en une hydrolyse par un mélange d'acides acétique, trichloracétique et nitrique pendant $30 \mathrm{mn}$; cette technique, dont les résultats sont comparables à la méthode de WEENDE, présente sur cette dernière de nets avantages de rapidité et de précision. L'amidon a été dosé par voie enzymatique (CHARLIER et al., I974). A partir des matières minérales totales, déterminées après calcination à $55^{\circ}{ }^{\circ} \mathrm{C}$, on a également dosé le calcium (par photométrie de flamme) et le phosphore (par colorimétrie).

L'évolution de la glycémie et celle de la lactacidémie sont suivies à partir d'échantillons de sang prélevés juste avant le repas du matin puis, à nouveau, $2 \mathrm{~h}$, $4 \mathrm{~h}$ et $6 \mathrm{~h}$ après; nous indiquons les moyennes des résultats obtenus pendant 2 jours consécutifs de prélèvements. Le glucose est dosé par colorimétrie (réaction avec l'orthotoluidine, technique bio-Mérieux) et l'acide lactique par une méthode enzymatique à la lacticodéshydrogénase (réactifs bio-Mérieux).

\section{B. - Essais sur poneys fistulés}

\section{I. -- Animanx et aliments.}

Trois poneys sont munis d'une canule au niveau du cæcum, la fistulation a été réalisée par $D$. Gouy selon une technique dérivée de celle de TEETER, NeL,SON et STILIIONS (I968).

Ces animaux reçoivent $2 \mathrm{~kg} / \mathrm{j}$ de l'un des aliments complets marqués avec 
0,2 p. cent d'oxyde de chrome. La ration est fractionnée en 2 repas distribués à 8 et $20 \mathrm{~h}$; durant la période d'adaptation de 3 semaines chaque poney est entraîné à consommer sa ration en un temps limité à I heure.

\section{2. - Techniques de laboratoire.}

a) La digestibilité totale a été évaluée à l'aide des marqueurs, à partir de prélèvements aliquotes de fèces récoltées à $8-\mathrm{I} 2$ et $\mathrm{I} 8 \mathrm{~h}$, pendant trois jours consécutifs. Les échantillons de la même heure sont rassemblés et analysés; comme il n'est pas apparu de différences en fonction de l'heure de récolte, les résultats rapportés sont les moyennes des 3 prélèvements quotidiens.

Parallèlement, chez ces mêmes poneys fistulés, au cours d'une période expérimentale faisant suite à la phase d'adaptation, des prélèvements du contenu cæecal global (solide et liquide) sont réalisés toutes les 2 heures pendant $\mathrm{I} 2$ heures à partir de la distribution du repas. Après séchage à $80^{\circ} \mathrm{C}$, afin d'évaluer la matière sèche, les matières azotées totales et la cellulose brute sont dosées et les coefficients d'utilisation digestive partiels sont calculés par référence, soit au marqueur incorporé (oxyde de chrome, dosé par absorption atomique), soit à la lignine, utilisée comme marqueur naturel et dosée selon la méthode de VAN SoEsT (Ig63).

b) Les prélèvements de liquide cxeal ( sont effectués au moyen d'une sonde introduite par la canule et plongeant dans le cæcum, munie à son extrémité distale d'un sac en toile de nylon faisant filtre à l'encontre des particules solides. Après la période d'adaptation, ces prélèvements sont renouvelés toutes les heures pendant i I $h$ successives (en commençant juste avant la distribution du repas du matin), au cours de 3 séries répétées à 2 jours d'intervalle.

Sur les échantillons de jus de cæcum, sont déterminés : le pH (au pH mètre), l'ammoniac (dosé à l'autoanalyseur Technicon par la réaction de BERTHELOT, après dilution au I/4 dans l'acide trichloracétique $2,5 \mathrm{p}$. cent, puis au I/Io dans l'eau, selon la technique exposée par Michet., I97I), les acides gras volatils (par chromatographie en phase gazeuse, après reprise par l'acide phosphorique de l'échantillon congelé), et l'acide lactique (par voie enzymatique à la lacticodéshydrogénase, après dilution du prélèvement au I/3 par l'acide perchlorique $0,6 \mathrm{~N}$, en utilisant les réactifs Bio-Mérieux).

\section{Résultats}

\section{A. - Mesures sur les 6 poneys en cages à métabolisme}

I. - Les moyennes des digestibilités apparentes totales sont réunies dans le tableau 2 .

2. - L'évolution des glycémies et des lactacidémies est rapportée dans le tableau 3 pour ces deux paramètres, il n'apparaît pas de différence significative en fonction des trois présentations de l'aliment. 


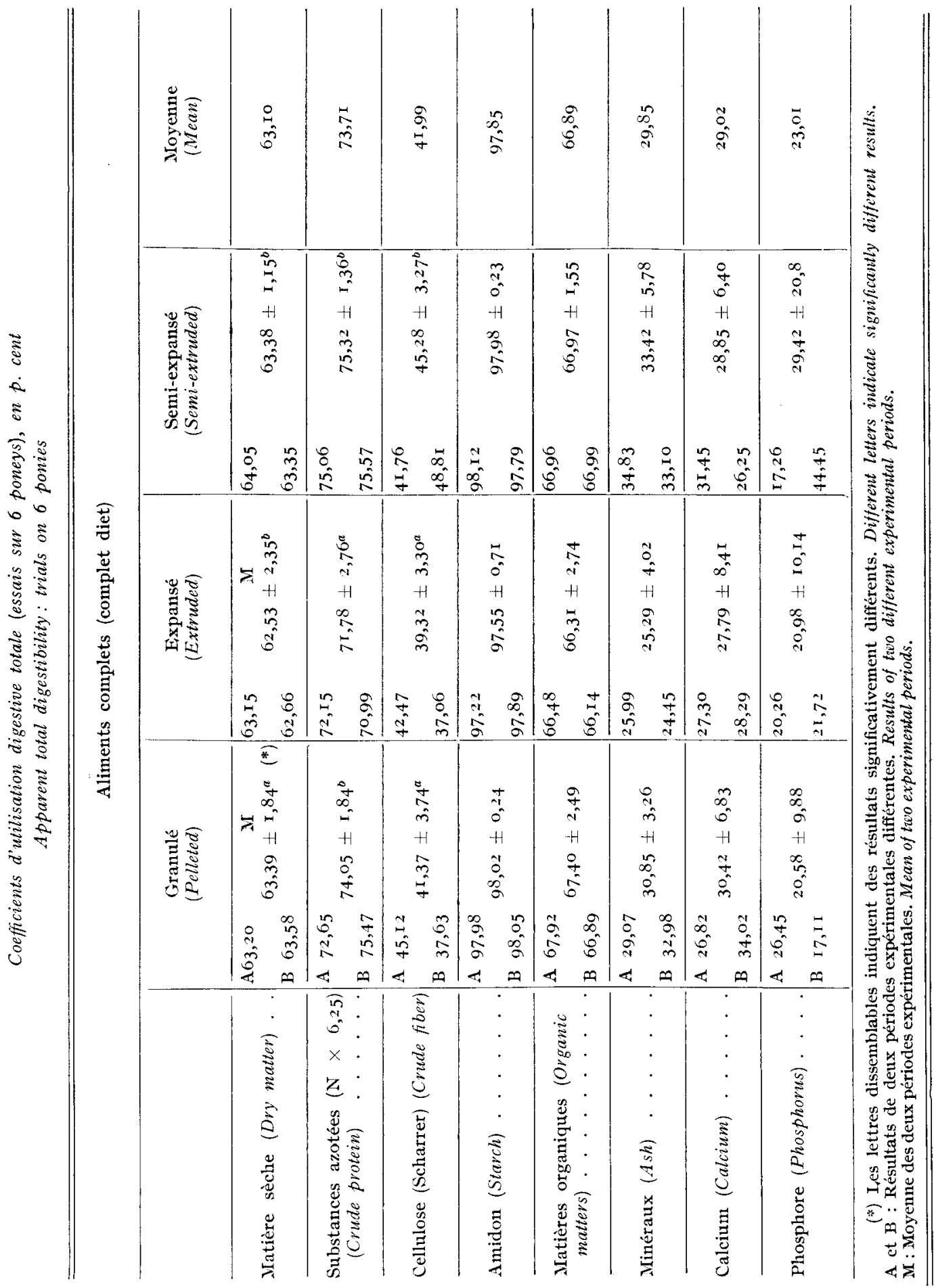




\section{TABLEAU 3}

Évolution des taux plasmatiques de glucose et d'acide lactique

Kinetics of blood glucose and lactate levels

\begin{tabular}{|c|c|c|c|c|c|c|c|c|}
\hline & \multicolumn{4}{|c|}{$\begin{array}{l}\text { Glycémie }(\mathrm{g} / \mathrm{l}) \\
(\text { Glycemia })\end{array}$} & \multicolumn{4}{|c|}{$\begin{array}{c}\text { Lactacidémie }(\mathrm{mg} / \mathbf{1}) \\
(\text { Lactacidemia) }\end{array}$} \\
\hline & $\begin{array}{l}\text { Granulé } \\
\text { (Pellet- } \\
\text { ed })\end{array}$ & $\begin{array}{c}\text { Expansé } \\
(E x t r u d- \\
e d)\end{array}$ & $\begin{array}{c}\text { Semi- } \\
\text { Expansé } \\
(\text { Semi- } \\
\text { extruded })\end{array}$ & $\begin{array}{c}\text { Moyenne } \\
(\text { Mean })\end{array}$ & $\begin{array}{l}\text { Granulé } \\
(\text { Pellet- } \\
e d)\end{array}$ & $\begin{array}{c}\text { Expansé } \\
(\text { Extrud } \\
e d)\end{array}$ & $\begin{array}{c}\text { Semi- } \\
\text { Expansé } \\
\text { (Semi- } \\
\text { extruded })\end{array}$ & $\begin{array}{l}\text { Moyenne } \\
(\text { Mean })\end{array}$ \\
\hline to $\quad=\begin{aligned} & \text { repas } \\
& (\text { Meal })\end{aligned}$ & 0,86 & 0,85 & 0,83 & 0,85 & $5^{8,9}$ & 50,0 & $5 \mathrm{I}, 3$ & 53,4 \\
\hline to +2 heures (hours) & $\mathrm{I}, 32$ & $\mathbf{I}, 23$ & I, 26 & $\mathbf{I}, 27$ & 60,7 & $7 x, 3$ & 66,5 & 66,2 \\
\hline to +4 heures. & I,o8 & I, 06 & $\mathbf{I}, 00$ & $\mathrm{I}, 04$ & 71,7 & 62,2 & 72,0 & 68,6 \\
\hline to $f-7$ heures & 0,98 & 0,95 & $0,9 I$ & 0,95 & 62,3 & $6 \mathrm{I}, 2$ & 59,7 & $6 \mathrm{r}, \mathrm{r}$ \\
\hline
\end{tabular}

\section{TABLEAU 4}

Digestibilités totales moyennes chez 3 poneys porteurs de canules cacales (en p.cent)

Mean values of total digestibilities in 3 fistulated ponies

\begin{tabular}{|c|c|c|c|c|c|c|}
\hline & & & $\begin{array}{l}\text { Granulé } \\
(\text { Pelleted })\end{array}$ & $\begin{array}{c}\text { Expansé }\left(^{*}\right) \\
(\text { Extruded })\end{array}$ & $\begin{array}{l}\text { Semi-expansé } \\
(\text { Semi-extruded })\end{array}$ & $\begin{array}{l}\text { Moyenne } \\
(\text { Mean })\end{array}$ \\
\hline $\begin{array}{l}\text { Matière sèche } \\
\text { (Dry matter) }\end{array}$ & $\begin{array}{l}A \\
B \\
C\end{array}$ & $\begin{array}{l}\cdot \cdot \cdot \\
\cdot \cdot \cdot \\
\cdot \quad \cdot\end{array}$ & $\begin{array}{l}62,60 \pm 3,05 \\
61,01 \pm 3,77 \\
51,60 \pm 3,93\end{array}$ & $\begin{array}{l}52,90 \pm 7,54 \\
53,32 \pm 4,01 \\
49,10 \pm 6,53\end{array}$ & $\begin{array}{l}63,00 \pm 6,84 \\
62,78 \pm I, 58 \\
55,90=10,04\end{array}$ & $\begin{array}{l}59,50 \\
59,03 \\
52,20\end{array}$ \\
\hline $\begin{array}{l}\text { Substances } \\
\text { azotées }(\mathrm{N} \times 6,25) \\
\quad(\text { Crude protein })\end{array}$ & $\begin{array}{l}A \\
B \\
C\end{array}$ & $\begin{array}{l}\cdot \cdot \cdot \\
\cdot \quad \cdot \\
\cdot \quad \cdot\end{array}$ & $\begin{array}{l}68,30 \pm 4,76 \\
68,53 \pm 4,29 \\
61,57 \pm 5,31\end{array}$ & $\begin{array}{l}67,10 \div 3,42 \\
67,21 \div 0,34 \\
64,80 \pm 0,71\end{array}$ & $\begin{array}{l}69,96 \pm 5,81 \\
69,98 \pm 2,36 \\
65,90 \pm 8,59\end{array}$ & $\begin{array}{l}68,45 \\
68,57 \\
64,09\end{array}$ \\
\hline $\begin{array}{l}\text { Cellulose (de scharrer) } \\
\qquad \text { (Crude fiber) }\end{array}$ & $\begin{array}{l}\text { A } \\
\mathbf{B} \\
\mathbf{C}\end{array}$ & 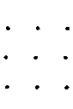 & $\begin{array}{l}48,78 \pm 7,70 \\
45,10 \pm 3,97 \\
31,80 \pm 1,48\end{array}$ & $\begin{array}{l}41,96 \pm 5, \text { I I } \\
46,96 \div 0,76 \\
36,55 \div 5,02\end{array}$ & $\begin{array}{l}49,7^{2} \pm 5,8 \mathrm{I} \\
5 \mathrm{I}, 54 \pm 9,40 \\
37, \mathrm{IO} \pm 7,5 \mathrm{I}\end{array}$ & $\begin{array}{l}46,79 \\
47,86 \\
35,13\end{array}$ \\
\hline
\end{tabular}

A : Récolte totale des matières fécales. Total collection of faeces.

B : Avec la lignine de Van Soest comme traceur. With Van Soest lignin as a marker.

C : Par marquage à l'oxyde de chrome. With chromic oxide as a marker.

(*) I,es moyennes concernant cette présentation n'ont pu ètre faites que sur 2 animaux, le troisième ayant éte malade. For this diet, means are obtained with two animals only, because of the sickness of the third one. 


\section{B. - Mesures sur les poneys fistulés}

I. - Digestibilités, totale et partielle (au niveau du cæcum)

A propos des mesures de digestibilités totales (jugées au niveau des matières fécales), la comparaison de 3 méthodes, soit par récolte complète, soit par référence à la lignine (dosée par la méthode de Van Soest), soit par marquage à l'oxyde de chrome, montre une très bonne corrélation entre les deux premières techniques, alors que la troisième fournit des résultats systématiquement inférieurs, comme le montre le tableau 4.

De ce fait, les digestibilités cæcales, récapitulées au tableau 5 , ont été calculées en utilisant la lignine comme traceur.

TABLEAU 5

Digestibilités partielles cacales (moyenne de 3 poneys)

Partial cacal digestibility: mean of 3 ponies

\begin{tabular}{|c|c|c|c|c|}
\hline P. cent & $\begin{array}{l}\text { Granulé } \\
(\text { Pelleted })\end{array}$ & $\begin{array}{c}\text { Expansé } \\
\text { (Extruded) }\end{array}$ & $\begin{array}{l}\text { Semi-expansé } \\
\text { (Semi-extruded) }\end{array}$ & $\begin{array}{l}\text { Moyenne } \\
(\text { Mean })\end{array}$ \\
\hline $\begin{array}{l}\text { Matière sèche } \\
\text { (Dry mattcr }\end{array}$ & $53,09=4,19$ & $41,68 \therefore 6,4^{2}$ & $49,4 \mathrm{I}: 5, \mathrm{I} 4$ & $\begin{array}{c}4^{8,06} \\
(81,4 \text { p. cent })\left(^{*}\right)\end{array}$ \\
\hline $\begin{array}{l}\text { Matières protéiques } \\
\text { (Crude protein). . }\end{array}$ & $53,36 \ldots 10,89$ & $55,73 \div 2,73$ & $52,24=1 \mathrm{I}, \mathrm{I} 9$ & $\begin{array}{c}53,44 \\
(77,9 \text { p. cent })(*)\end{array}$ \\
\hline $\begin{array}{l}\text { Matières cellulosiques } \\
\text { (Crude fiber). . . }\end{array}$ & $37,12 \doteq$ I I , OO & $29,63 \div 3,06$ & $23,7 x+4,5^{2}$ & $\begin{array}{c}3^{0,15} \\
(62,9 \text { p. cent })(*)\end{array}$ \\
\hline
\end{tabular}

$\left(^{*}\right)$ Pourcentage calculé par rapport à la digestibilité totale. Percentage calculatcd in relation to total digestibility.

\section{TABLEAU 6}

Évolution du taux de matière sèche dans le cacum (p. cent)

l'ariations of d'y matter level in the cacum ( $p$. cent)

\begin{tabular}{|c|c|c|c|c|c|}
\hline & & $\begin{array}{l}\text { Granulé } \\
\text { (Pelleted })\end{array}$ & $\begin{array}{c}\text { Expansé } \\
\text { (Extruded) }\end{array}$ & $\begin{array}{c}\text { Semi-expansé } \\
\text { (Semi-extruded) }\end{array}$ & $\begin{array}{c}\text { Moyenne } \\
\text { (Mean) }\end{array}$ \\
\hline to $=\operatorname{repas}($ Meal $)$ & . . . . & $6,39 \pm 3,20$ & $7,41 \div 4,29$ & $6,45=1,76$ & 6,75 \\
\hline to + I heure (Hour) & . & $4,49 \pm 2,03$ & $6,23 \div 3,77$ & $5,69 \pm 1,79$ & 5,47 \\
\hline to +2 heures ... & & $5,31 \pm 2,18$ & $6,12 \cdots 3,54$ & $4,72=2,26$ & 5,38 \\
\hline to +3 heures & . & $7,85 \pm 2,21$ & $7,62 \div 1,23$ & $6,79=2,40$ & 7,42 \\
\hline to +5 heures & & $9,33 \pm 2,02$ & $8,87=\mathbf{I}, 63$ & $10,01 \quad I, 59$ & $9,4^{\circ}$ \\
\hline to +7 heures. & 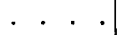 & 9, I I $\pm 2,44$ & $7,50=0,22$ & $9,88 \pm 0,61$ & 8,83 \\
\hline to +9 heures . . & . & $8,04 \pm \mathrm{I}, 88$ & $7,73 \div 0,99$ & $\mathrm{I} I, 55 \div \mathrm{I}, 57$ & 9,10 \\
\hline to $f$ II heures. & 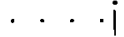 & $9,47 \pm 3,60$ & $6,01 \div 1,47$ & $9,20 \pm 2,27$ & 8,22 \\
\hline
\end{tabular}


2. - Évolution de la composition du liquide cacal

L'eau représente 90 à 95 p. cent du contenu du cæcum, avec un minimum entre 5 et $9 \mathrm{~h}$ après le repas (comme cela apparaît au tabl. 6).

- Le $p H$ qui est proche de 7 au moment du repas, s'abaisse vers 6,8 entre 5 à $8 \mathrm{~h}$ suivant celui-ci, donc de façon synchrone avec l'augmentation de la teneur en matière sèche (fig. I).

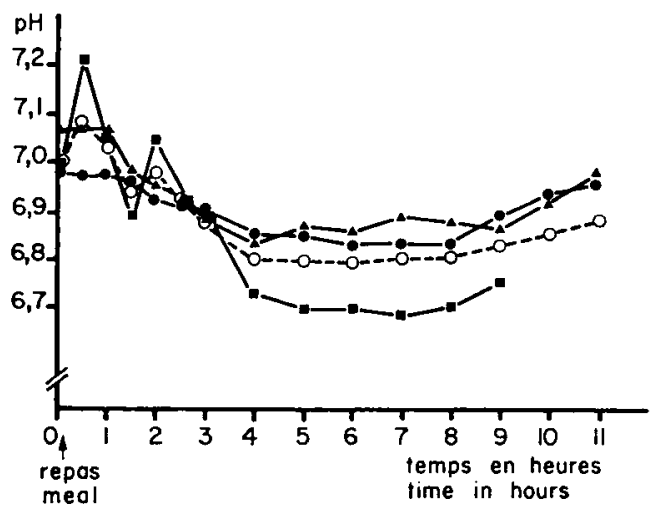

FIG. I. - Cinétique du $p H$ dans le cacum. Evolution of cacal fuid $p H$.
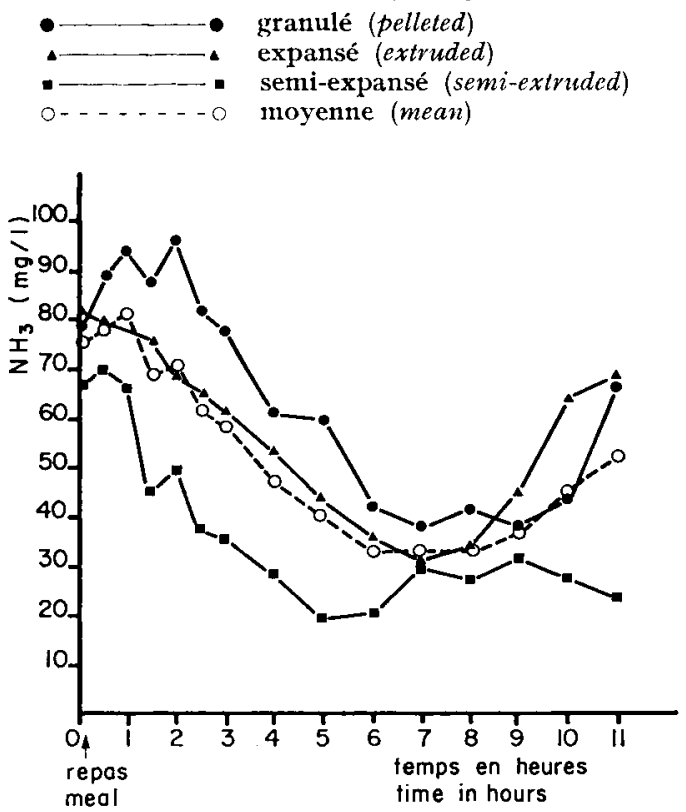

FIG. 2. -- Cinétique de l'ammoniac dans le cacum. Evolution of cecal fuid ammonia concentration.

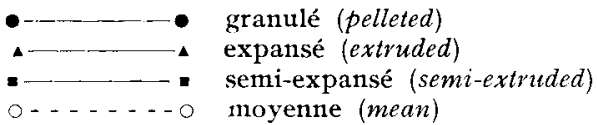




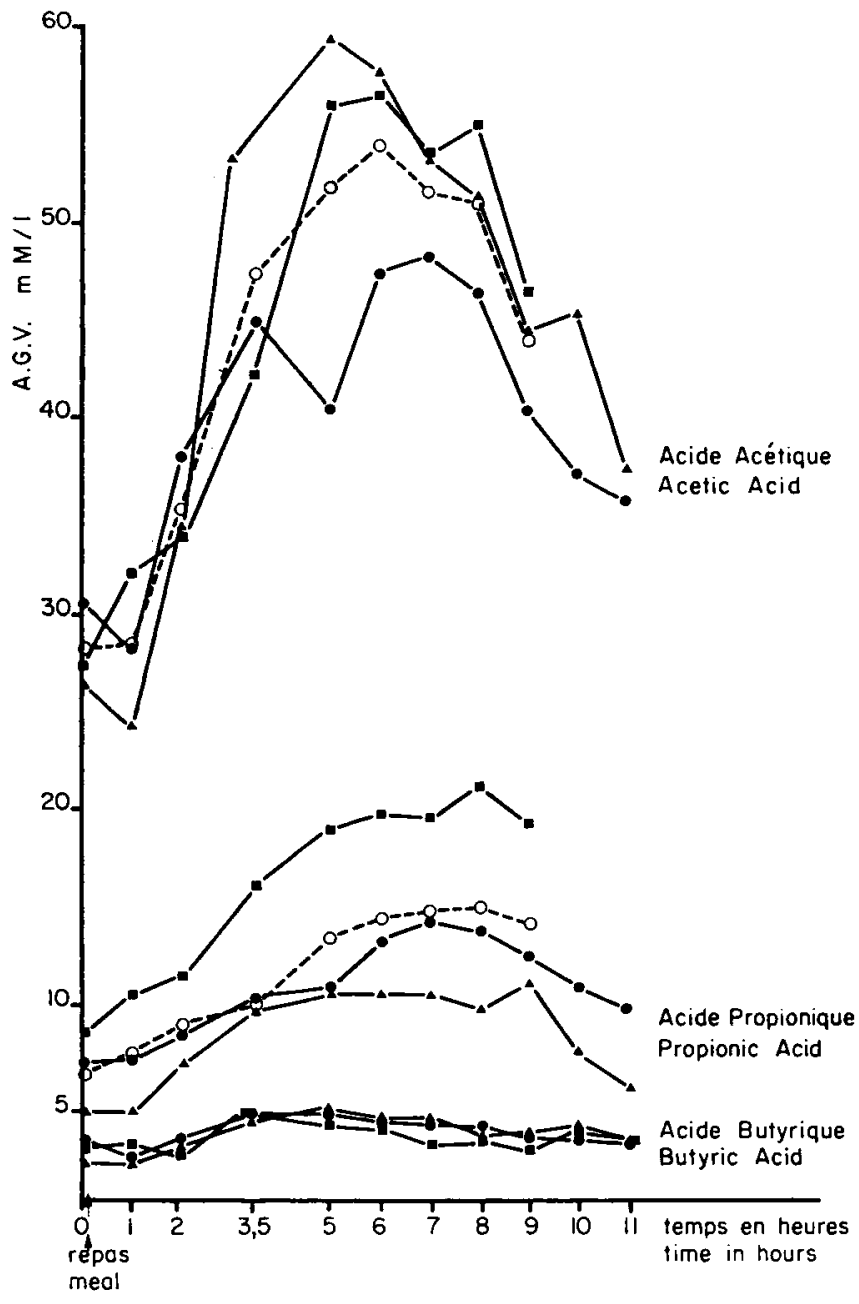

Fig. 3. - Cinétique des A.G.V. dans le cacum. Evolution of VFA concentration in cacal fuid.

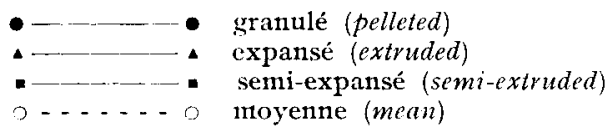

- Le taux d'ammoniac suit une courbe similaire bien que plus accentuée; de $80 \mathrm{mg} / 1$ chez l'individu à jeun, il passe par un minimum de l'ordre de 20 à $40 \mathrm{mg} / 1$, de 5 à $9 \mathrm{~h}$ après le repas (fig. 2).

- Les concentrations en acides gras volatils (AGV) évoluent bien en sens inverse du $\mathrm{pH}$, démontrant ainsi leur rôle primordial sur celui-ci. Minimales au départ, elles atteignent un maximum 5 à $9 \mathrm{~h}$ après le repas, sans que soient sensiblement affectées les proportions relatives des acides acétique, propionique et butyrique, comme l'indiquent la figure 3 et le tableau 7 . 


\section{TABLEAU 7}

$p H$, taux d'ammoniac, d'AGV et pourcentages d'A $G V$ Geures après le repas $p H$, ammonia, VFA concentration and VFA percentage 6 hours after feeding

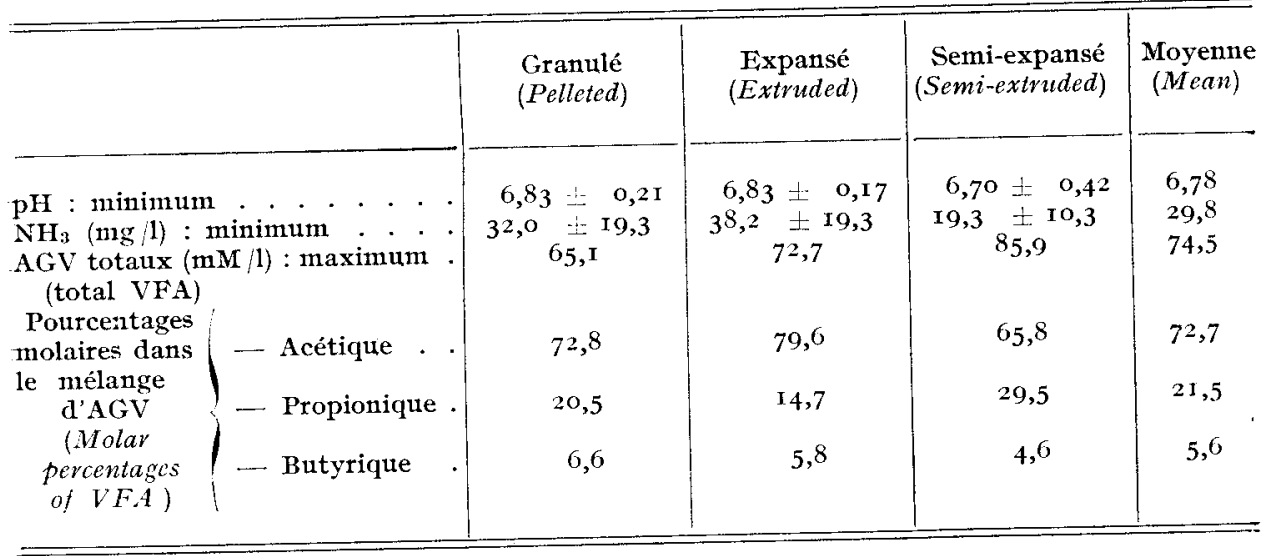

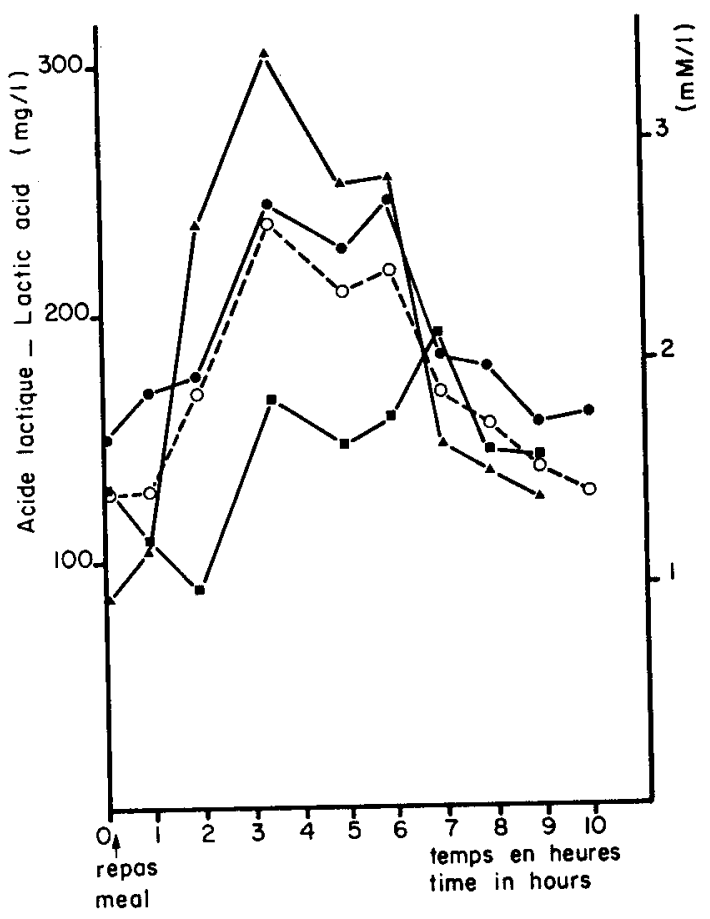

FIG. 4. - Cinétique de l'acide lactique dans le cacum. Evolution of lactic acid concentration in cacal fuid.

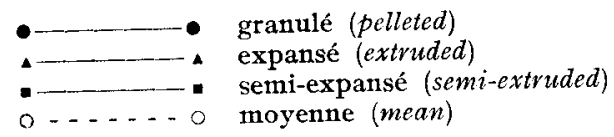


- Les teneurs en acide lactique restent très basses, avec un léger pic à $3 \mathrm{mM} / 1$ environ 3 à $5 \mathrm{~h}$ après le repas, donc un peu plus précoce que celui des acides gras volatils (fig. 4).

\section{Discussion}

\section{A. - Digestibilités}

Dans le cas d'une alimentation strictement restreinte et fragmentée, le conditionnement de l'aliment a peu d'influence sur la digestibilité des constituants de 1a ration. Bien que l'utilisation digestive de la matière sèche paraisse meilleure pour le granulé que pour les deux autres présentations, l'expansion peut accroître de façon faiblement significative la digestibilité de la cellulose : 45,3 p. cent (granulé : 4I,4 p. cent). Pour les matières azotées, leur digestibilité est significativement amoindrie par l'expansion : 71,8 p. cent (granulé : 74 p. cent; semi-expansé : 75,3 p. cent). Ces résultats sont comparables à ceux obtenus lors d'un précédent essai (Wolter, Derix et Letournead, 1976).

L'attaque enzymatique précoce et puissante de l'amidon au niveau de 1 'in. testin grêle en explique la digestion quasi-totale, en moyenne de 97,4 p. cent. Toutefois, il a été vérifié au cours d'essais pratiques sur chevaux (WOLTER et al., I977), que la valeur énergétique de l'aliment complet était nettement améliorée par l'expansion; il faut alors supposer que le traitement hydrothermique entraînant une gélatinisation partielle de l'amidon faciliterait ainsi la digestion enzymatique dans l'intestin grêle, et favoriserait la résorption du glucose dont le rendement énergétique est meilleur que celui des acides gras volatils qui seraient libérés dans le gros intestin.

Cependant, dans le cas présent, il n'apparait pas de variation de la glycémie susceptible de confirmer l'hypothèse précédente, mais il est vrai que la régulation hormonale de cette glycémie est excellente chez les équidés (Hintz, ARGENzio et SChRYVER, I97I).

Par ailleurs, la confrontation des tableaux 2 et 4 laisserait penser que le port de canules permanentes du cæcum est susceptible de retentir quelque peu sur la digestibilité (estimée par la méthode de récolte totale). Toutefois les résultats ne sont pas univoques puisque la digestibilité de la matière sèche $(59,5 \mathrm{~F}$. cent au lieu de $64 \mathrm{p}$. cent) et celle des substances azotées $(68,4 \mathrm{p}$. cent au lieu de 73,7 p. cent) tendent à baisser alors que celle de la cellulose serait plutôt améliorée (46,8 $\mathrm{p}$. cent au lieu de $4^{2} \mathrm{p}$. cent), peut-être en raison d'un ralentissement du transit au niveau du gros intestin.

\section{B. - Digestibilité partielle cuecale}

La présentation de l'aliment ne modifie pas significativement les digestibilités partielles de la matière sèche, des matières azotées ou des matières cellulosiques (tab1. 5). Les écarts-types souvent ćlevés traduisent une variabilité individuelle particulièrement importante.

La proportion de matière sèche digérée au niveau du cæcum atteint $80 \mathrm{p}$. cent de la matière sèche digestible totale. Ce pourcentage est comparable à celui constaté par Hintz et al. (I97I) chez des chevaux recevant un régime riche en céréales. 
Toutefois, cette valeur est un peu plus élevée que celle observée ( $65 \mathrm{p}$. cent) chez des chevaux alimentés avec un aliment complet et abattus $3 \mathrm{~h}$ après le repas (Wolter et Gouy, I976).

Confirmant également que l'intestin grêle joue un rôle majeur dans la digestion des protéines (Reirnour et al., I970; Wolter et Víl.andia, I970), cet essai met en évidence que près de $8 \mathrm{o}$ p. cent des matières azotées digestibles ont déjà disparu au niveau du cxcum. Par ailleurs, rappelons que Rirtrour et SALSBURY (I972) estiment que la resynthèse microbienne dans le gros intestin ne serait d'aucun profit chez le cheval, car la résorption azotée ne se ferait alors que sous forme d'ammoniac.

La part de la cellulose digestible dégradée au niveau du cæcum atteint déjà 63 p. cent, ce qui confirme le rôle important de la digestion cxcale des glucides membranaires, dont une portion non négligeable sera encore attaquée dans le côlon. Cependant, ce chiffre qui semble relativement élevé quand on sait que la cellulolyse ne commence que dans le crecum (WoLter et Gour, I976) s'explique vraisemblablement dans les conditions de cet essai par le faible taux de cellulose de l'aliment (environ I6,5 p. cent) et par le broyage préalable qui facilite les fermentations microbiennes précoces, même si l'accélération du transit digestif qui en résulte tend plutôt à réduire la digestibilité finale de la cellulose.

\section{C. - Activité biochimique du cacum}

Dans nos conditions expérimentales, la composition du contenu cæcal n'est pas significativement in fluencée par la présentation de l'aliment complet, mais elle suit, en fonction des repas une évolution apparemment très cohérente :

- Le pH, qui donne une bonne image synthétique des fermentations cæcales, s'abaisse assez peu à la suite des repas, montrant ainsi que dans le cas d'une alimentation restreinte, fractionnée et relativement concentrée, l'essentiel de la digestion se déroule dans l'intestin grêle et que ce 1:'est qu'un sulsstrat déjà largement épuisé qui atteint le gros intestin, de telle sorte que l'activité microbienne y est alors très tempérée.

D'ailleurs, le taux ammoniacal s'amenuise, laissant supposer qu'il procède davantage d'une autolyse des protéines microbiennes que d'une ammoniogenèse à partir des apports azotés alimentaires. A ce propos, Reirnour et SAIsBury (I975) montrent que la majorité des protéines contenues dans le cxcum est dégradée exi ammoniac avant d'être absorbée et ne contribue donc pas à la couverture des besoins en acides aminés indispensables.

Toutefois, il convient de remarquer que ce $\mathrm{pH}$ est légèrement diminué entre 5 et 8 heures après le repas; cette période correspond justement à 1'afflux de la phase particulaire des digesta, comme on peut le suspecter d'après l'augmentation de la teneur en matières sèches du contenu cæcal (tabl. 6) et comme nous l'avons vérifié au moyen de repas marqués avec des particules colorées.

De façon très parallèle, on constate une élévation des concentrations en acides gras volatils, traduisant une stimulation de l'activité microbienne au bénéfice de la cellulolyse. Malgré tout, les fermentations restent modérées conipte tenu de la faible abondance du substrat et de la disparition quasi totale des éléments les plus fermentescibles; dès lors, on comprend que le $\mathrm{pH}$ diminue peu et que les proportions des divers acides gras volatils dans le mélange total ne soient pas sensiblement modifiées au cours de la journée. Inversement, GLinsky et al. (I976) ont montré que la production creale d'acides gras volatils tend à augmen- 
ter avec la proportion de fourrages dans la ration, au plus grand profit de la fermentation acétique.

A plus forte raison, la teneur en acide lactique demeure très basse, et il n'est même pas exclu qu'une part provienne des segments antérieurs. Ce n'est donc qu'à l'occasion d'une surconsommation brutale et exagérée d'aliments amylacés, tels que des céréales, que l'afflux massif de glucides facilement fermentescibles dans le cæecum serait susceptible d'accélérer vivement l'acidification du contenu cæcal et d'induire une production notable d'acide lactique.

Accepté pour publication en octobre 1977.

\section{Summary}

\section{Digestibility and biochemical activity in the cacum of ponies receiving a diet of ordinary pellets, extruded or semi-extruded pellets}

The ponies were fed with ordinary pellets, extruded or semi-extruded pellets (moist - heat treatment of the cereal part only) containing $16,5 \mathrm{p}$. cent crude fibre, ro p. cent protein (Table I) and marked with $0,2 \mathrm{p}$. cent chromic oxide.

The experimental design was the following:

- Six ponies placed in digestibility cages received $3 \mathrm{~kg}$ feed/animal/ day in two meals.

- - Three ponies with permanent fistulae of the caecum received only $2 \mathrm{~kg}$ feed/animal/day in two meals of one hour each.

In these conditions of severely restricted feeding, of a rather concentrated diet distributed over two meals, the three physical forms of the feed did not lead to large differences either as regards the digestibility coefficient (Table 2), partial digestibility (Table 5) and metabolism in the cacum (Table 7) (measured by $\mathrm{pH}$, ammonia percentage, volatile fatty acids and lactic acid) (fig. I, 2, 3,4) or as regards glycemia and lactacidemia (table 3).

The digestibility measures show that use of a natural marker such as lignine, according to Van Soest's method, offers more reliability than the method using the chromic oxide marker (Table 4).

\section{Références bibliographiques}

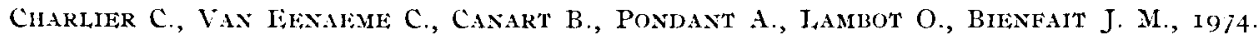
Méthode de dosage seini-automatique de l'amidon et du glucose dans les aliments du bétail. Ann. Med. vet., 118, 28I-294.

(ilinsky M. J., Simin R. M., SPIREs H. R., DAvis C. L., I977. Measurement of volatile fatty acid production rates in the cacum of the poney. J. Anim. Sci., 42, 1465-1470.

Crovy D., I976. Une étude de la digestion chez les équidés par analyse du contenu intestinal après abattage. Thise de doctorat vétérinaive, $\mathrm{I}$ yon.

Hint\% H. F., Argexzio R. A., Schrvvir H. F., i97 r. 1)igestion coefficient, blood glucose levels and inolar percentage of volatile fatty acids in intestinal fluid of ponies fed varying forage grain ratios. I. Anim. Sci, 33, $992-995$.

HiNTt H. F., WAlkir E. F., Lowe, J. E., Scimyver H. I'., 197 I. Apparent digestion in various segments of the digestive tract of ponies diets with varing roughage ratios. J. Anim. Sci., $32,245^{-24} 8$.

Mrché M. C., r97г. Analyse quantitative de quelques substances azotées et glucidiques en milieu biologique. Essai de rationalisation. Thèse de doctorat d'Université.

Riritiour C. M., Baker J. P., Mitcheld G. E., LitTle C. O., Kratzen D. D., I97o. Aminoacids in equine cxcal contents, cecal bacteria and serum. $J$. Nutr., 100, 349-354.

REIT,NOUR C. M., SALSBLRY R. L., I972. Digestion and utilization of caecally infused protein by the equine. J. Anim. Sci., 35, I I90-1 193 . 
Reitnour C. M., SAISBURy R. L., I975. Effect of oral or cæcal administration of protein supplements on equine plasma aminoacids. Brit. Vet. J., 131, 466-473.

SchARRER K., KURSChNeR K., I932. Ein neues, resch durchfürhares Verfahren 2. Beitimmung d. Rohfaser in Futtermitteln. Tievernährung, 3, 302 .

TEETER S. M., Nelson W. E., STritions M. C., 1968. Cæcal fistulation in the horse. J. Anim. Sci., 27, 394-404.

VAN SoEst J. P., I963. Use of detergents in the analysis of fibrous feed. II. A rapid method of the détermination of fiber and lignin. J. A.O.A.C., 46, 829-835.

Wol.ter R., Velandia J., I97o. Digestion des fourrages chez l'âne. Rec. Méd. Vét., 144, I 4 I-I 52 .

Wolter R., Durix (Andrée), LETourniau J. C., 1974. In fluence du mode de présentation du fourrage sur la vitesse du transit digestif chez le poney. Ann. Zootech., 23, 293-300.

WoI.TER R., DURIX (Andrée), Letourneau J. C., 1975. Influence du mode de présentation du fourrage sur la digestibilité chez le poney. Ann. Zootech., 24, 237-242.

Wotiter R., DURIX (Andrée), Iftrourneau J.C., I976. Influence du mode de présentation d'un aliment complet sur la vitesse du transit digestif et la digestibilité chez le poney. $A \mathrm{~m}$. Zootech., 25, $18 \mathrm{r}-188$.

WOLter R., Gouy D., 1976. Litude expérimentale de la digestion chez les équidés par analyse du contenu intestinal après abattage. Rev. Med. I'et., 127 (12), I 723-1 $73^{6}$.

Wol,Ter R., Meunifr B., de Fadcompret R., Andrée DURIX et Jacqueline I,ANDREaU, i977. Essai d'un aliment complet, granulé ou expansé, en conparaison avec le régime traditionnel chez des chevaux de sport. Rev. Méd. Vét., 128, 71-81. 